\title{
Integrated Analysis of MiRNA and Genes Associated with Meat Quality Reveals that Gga-MiR-140-5p Affects Intramuscular Fat Deposition in Chickens
}

\author{
Meng Zhang ${ }^{\mathrm{a}}$ Dong-Hua Lia Fang Lia Jun-Wei Sun ${ }^{\mathrm{a}} \quad$ Rui-Rui Jianga,b \\ Zhuan-Jian Li ${ }^{a, b}$ Rui-Li Han ${ }^{a, b}$ Guo-Xi Li,b Xiao-Jun Liu ${ }^{a, b}$ \\ Xiang-Tao Kang ${ }^{a, b}$ Gui-Rong Sun ${ }^{a, b}$ \\ ${ }^{a}$ College of Animal Science and Veterinary Medicine, Henan Agricultural University, Zhengzhou, ${ }^{b} H e n a n$ \\ Innovative Engineering Research Center of Poultry Germplasm Resource, Zhengzhou, China
}

\section{Key Words}

Chicken • Meat quality $\bullet$ MiRNAs $・$ Intramuscular fat $•$ Adipocyte differentiation

\begin{abstract}
Background/Aims: Poultry meat quality is affected by many factors, among which intramuscular fat (IMF) is predominant. IMF content affects the tenderness, juiciness, and flavor of chicken. An increasing number of studies are focusing on the functions of microRNAs (miRNAs) during the adipogenic process. However, little is known about miRNAs associated with poultry IMF deposition, especially intramuscular adipocyte differentiation. Methods: The IMF content of two physiological stages was measured, and miRNA-Seq and RNA-Seq data were integrated and analyzed. A chicken intramuscular adipocyte cell differentiation model was constructed. A luciferase reporter assay, miRNA overexpression, and Oil Red O staining were used to confirm the targets of gga-miR-140-5p. Results: Our results showed that latelaying-period hens, which had a higher IMF content, exhibited lower global expression levels of miRNAs than juvenile hens. A total of 104 differentially expressed (DE) miRNAs were identified between the two groups. Integrated analysis of differentially expressed genes and DE miRNAs identified a total of 378 miRNA-mRNA pairs. Functional enrichment analysis revealed that these intersecting genes are involved in ubiquitin-mediated proteolysis, the peroxisome proliferator-activated receptor signaling pathway, glycerophospholipid metabolism, and fatty acid elongation and degradation pathways. Furthermore, we demonstrated that gga-miR140-5p promoted intramuscular adipocyte differentiation via targeting retinoid $\mathrm{X}$ receptor gamma. Conclusion: Our findings may contribute to a more thorough understanding of chicken IMF deposition and the improvement of poultry meat quality.

M. Zhang, D.-H. Li and F. Li contributed equally to this work. 


\section{Cellular Physiology Cell Physiol Biochem 2018;46:2421-2433 \\ and Biochemistry Published onlıne: May 09, $2018 \quad \begin{aligned} & \text { DOI: 10.1159/000489649 } 2018 \text { The Author(s). Published by S. Karger AG, Basel } \\ & \text { www.karger.com/cpb }\end{aligned}$ \\ Zhang et al.: The Effection of miRNA on Chicken Intramuscular Fat Depositon}

\section{Introduction}

Poultry meat is one of the main protein sources in human diets. Over the past few years, meat quality has attracted much scientific attention [1-3]. Meat quality is affected by many factors, including intramuscular fat (IMF) content, tenderness, drip loss, $\mathrm{pH}$, color, and flavor. Many previous studies mainly focused on the effects of genetic differences between breeds [4-7]. However, the underlying molecular mechanisms related to meat quality among different physiological stages remain poorly understood.

MicroRNAs (miRNAs) are endogenous small non-coding RNAs (18-25 nucleotides) that posttranscriptionally regulate gene expression [8]. An increasing number of studies have demonstrated that miRNAs play important roles in various biological processes, such as cell proliferation [9], differentiation [10,11], apoptosis [12], metabolism, and diseases [13]. miR-133 and miR-206 are involved in skeletal muscle growth and development by repressing the expression of myogenic transcription factors, such as myogenic $M y o D, S R F$, and MEF2A $[10,14]$. Furthermore, several miRNAs (miR-27a/b, miR-122, miR-378, miR-143, and miR-144) associated with adipocyte differentiation and lipid metabolism were recently identified $[11,15]$. Previous studies suggested that miRNAs play crucial roles in animal meat quality $[16,17]$. In our previous study, we found a remarkable difference in the meat traits and lipid metabolism levels between juvenile and late-laying-period hens [18]. Furthermore, we identified numerous differentially methylated genes and differentially expressed genes (DEGs) associated with meat quality in breast muscle between the two physiological stages [18]. Given that there existed a considerable quantity of DEGs between the juvenile (20 weeks old) and late-laying-period (55 weeks old) hens and tremendous differences in meat quality and lipid metabolism levels, we speculated that miRNAs might serve as important regulators of chicken meat quality between different age stages.

The objective of this study was to identify miRNAs affecting the differences in meat quality between the two age stages of laying hens. Thus, breast muscle tissues of 20- and 55 -week-old hens were used for high-throughput sequencing. Furthermore, an integrated analysis of differentially expressed (DE) miRNAs and mRNAs was performed to elucidate the regulatory patterns of miRNAs and their network with putative target genes associated with meat quality in this research. Subsequently, based on combined analysis of expression profiles of miRNAs and potential target mRNAs, the candidate miRNAs involved in intramuscular adipocyte differentiation were further characterized. Our results may contribute to a better understanding of chicken IMF deposition and the improvement of the meat quality of poultry.

\section{Materials and Methods}

\section{Ethics statement}

All of the animal experiments were performed in accordance with the protocols approved by the Institutional Animal Care and Use Committee (IACUC) of Henan Agriculture University (Henan, China) (Permit Number: 11-0085; Date: 06-2011)

\section{Sample collection and RNA extraction}

Six Gushi hens were divided into two groups: G20W (20-week-old juveniles) and G55W (55-weekold late-laying-period hens). All birds were raised in the same environmental conditions with ad libitum water and food. Chickens were weighed and then killed by stunning and exsanguination $12 \mathrm{~h}$ after feed was withheld. Tissue samples were harvested after peeling the skin tissues (without using scalding water). The entire right breast was collected and stored at $-20{ }^{\circ} \mathrm{C}$ for trait measurements. Tissues including left breast muscle, liver, heart, spleen, lung, kidney, duodenum, ovary, and abdominal fat were immediately collected, snap-frozen in liquid nitrogen, and stored at $-80{ }^{\circ} \mathrm{C}$ until RNA extraction. Total RNA of each sample was extracted with TRIzol reagent (TaKaRa, Dalian, China) according to the manufacturer's protocols. RNA was quantified by Qubit 2.0 (Thermo Fisher Scientific, Waltham, MA, USA) and Nanodrop ND-2000 spectrophotometer (Thermo Fisher Scientific). RNA purity was assessed using an Agilent 2100 Bioanalyzer (Agilent, Santa Clara, CA, USA) with RNA integrity number $>8$. Total RNA was stored at $-80{ }^{\circ} \mathrm{C}$ until used. 


\section{Cellular Physiology Cell Physiol Biochem 2018;46:2421-2433 and Biochemistry Published online: May 09, $2018 \quad \begin{aligned} & \text { DOI: 10.1159/000489649 } 2018 \text { The Author(s). Published by S. Karger AG, Basel } \\ & \text { www.karger.com/cpb }\end{aligned}$ \\ Zhang et al.: The Effection of miRNA on Chicken Intramuscular Fat Depositon}

\section{Small RNA library construction and RNA sequencing}

A total of $1.5 \mu \mathrm{g}$ RNA from each sample was used to prepare the miRNA sequencing library as described in the Illumina ${ }^{\circledR}$ Small RNA Sample Prep Kit (NEB, Ipswich, MA, USA) protocol. Briefly, T4 RNA ligase 1 and T4 RNA ligase 2 (truncated) were used to ligate adapters to the $3^{\prime}$ and $5^{\prime}$ ends of RNA. cDNA was then synthesized with an RT primer, followed by PCR amplification. Subsequently, the amplification products were purified from agarose gels to construct the small RNA library. The concentration of the library was determined using Qubit 2.0, the library concentration was diluted to $1 \mathrm{ng} / \mu \mathrm{L}$, the insert size was detected using an Agilent 2100 Bioanalyzer (Agilent, Santa Clara, CA, USA), and the concentration of the library was quantified by quantitative PCR. The six miRNA libraries were sequenced using an Illumina HiSeq 2500 at Biomarker Technologies (Beijing, China).

\section{Data analyses}

The raw reads were filtered using the Illumina Pipeline filter (Solexa v0.3) to removed low-quality reads, adapter dimers, reads smaller than $18 \mathrm{nt}$ or longer than $30 \mathrm{nt}$, and common RNA families (rRNA, tRNA, snRNA, snoRNA) and repeats. The clean data for each sample, which were greater than 12.70 million reads, were compared with the known miRNA database in miRBase (v21) (http://www.mirbase.org/) to identify mature miRNAs or pre-miRNAs [19]. Furthermore, novel miRNAs were predicted by the miRDeep2 program [20], and RNA-fold software (http://rna.tbi.univie.ac.at/cgi-bin/RNAWebSuite/RNAfold.cgi) was used to analyze their structures.

\section{Differential expression analyses}

The expression levels of miRNAs in each sample were calculated and normalized by the transcripts per million clean reads (TPM) algorithm [21]. The DEGSeq R program was used to analyze the DE miRNAs [22]. miRNAs with $\mid \log 2$ (fold change) $\mid \geq 2$, false discovery rate $\leq 0.05$ were identified as DE miRNAs.

\section{miRNA target gene prediction, network interactions, and functional analyses}

Based on the sequences of the miRNAs (known and novel miRNAs), the targets of miRNAs and DE miRNAs were predicted using miRanda and RNAhybrid. Gene Ontology (GO) and Kyoto Encyclopedia of Genes and Genomes (KEGG) pathway enrichment of target genes and target DEGs were analyzed using the DAVID database (http://david.abcc.ncifcrf.gov/) [23]. The network interactions between miRNAs and mRNAs were constructed using Cytoscape software [24].

\section{Vector construction}

The $3^{\prime}$ untranslated region (UTR) of the retinoid X receptor gamma (RXRG) gene containing gga-miR140-5p binding sites was amplified from chicken genomic DNA by PCR, and subcloned into the XhoI-NotI site of the psiCHECK-2 vector (Promega, Madison, WI, USA) (hereafter referred to as RXRG-UTR-WT). A mutant RXRG $3^{\prime}$ UTR reporter was generated by mutating the seed region of the gga-miR-140-5p binding sites by overlap extension PCR (RXRG-UTR-Mut). Plasmid DNA was sequenced by Sangon Biotech (Shanghai, China) and extracted using an EndoFree Maxi Plasmid Kit (TIANGEN, Beijing, China).

\section{Luciferase reporter assay}

Luciferase reporter experiments were performed in DF1 (chicken embryo fibroblasts) cells. Cells were seeded in 6-well plates at a density of $5 \times 10^{5}$ cells/well and cultured under routine conditions with $10 \%$ fetal bovine serum (FBS). When the cells reached $70 \%$ to $80 \%$ confluence, $R X R G$-UTR-WT or RXRG-UTR-Mut (100 ng) was cotransfected with $50 \mathrm{nM}$ negative control or gga-miR-140-5p mimic (GenePharma, Shanghai, China) using $3 \mu \mathrm{L}$ of Lipofectamine 2000 (Invitrogen, Carlsbad, CA, USA) according to the manufacturer's instructions, and the medium was replaced $6 \mathrm{~h}$ later. The relative luciferase activity was measured $48 \mathrm{~h}$ after transfection by the Dual-Luciferase Reporter Assay System (Promega) on a Fluoroskan Ascent FL instrument (Thermo Fisher Scientific, Shanghai, China). Renilla luciferase activity was normalized to firefly luciferase activity. Each cotransfection was performed in 6 replicates.

Primary chicken intramuscular preadipocyte isolation, culture, and induction of differentiation

Intramuscular preadipocytes were isolated from two-week-old chickens using the method described by Zhang et al. [25]., with some modifications. In brief, Gushi chickens were provided by Sungo, Ltd. (Henan, 


\section{Cellular Physiology Cell Physiol Biochem 2018;46:2421-2433

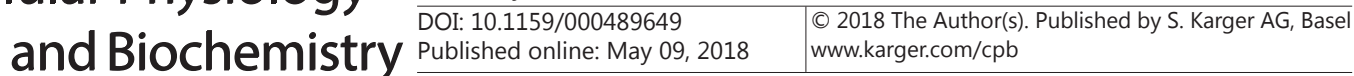 \\ Zhang et al.: The Effection of miRNA on Chicken Intramuscular Fat Depositon}

China). Breast muscle tissues were collected from 14-day-old Gushi chickens under sterile conditions, and then tissues were washed using phosphate-buffered saline (PBS) supplemented with penicillin (100 units/ $\mathrm{mL})$ and streptomycin $(100 \mu \mathrm{g} / \mathrm{mL})$. The washed tissue was cut into $1-\mathrm{mm}^{3}$ pieces using surgical scissors and then digested using $1 \mathrm{mg} / \mathrm{mL}$ collagenase type II (Solarbio, Beijing, China). The digested cell suspension was filtered using 200- and 500-mesh screens with gentle shaking at $37{ }^{\circ} \mathrm{C}$ for 90 min to separate the stromal-vascular fraction from undigested tissue and mature adipocytes, and then centrifuged at 1, $500 \mathrm{rpm}$ for $10 \mathrm{~min}$. Stromal-vascular cells were plated onto a $60-\mathrm{mm}$ culture plate at a density of $1 \times 10^{5}$ cells $/ \mathrm{mL}$. Cells were maintained in Dulbecco's modified Eagle's medium/Ham's nutrient mixture F-12 supplemented with 10\% FBS (Gibco, Beijing, China), 100 units/mL penicillin, and $100 \mu \mathrm{g} / \mathrm{mL}$ streptomycin in a humidified atmosphere with $5 \%(\mathrm{v} / \mathrm{v}) \mathrm{CO}_{2}$ at $37{ }^{\circ} \mathrm{C}$ until reaching $80-90 \%$ confluence. For the intramuscular adipocyte differentiation, upon reaching confluence, cells were exposed to differentiation medium consisting of base medium supplemented with $50 \mathrm{nM}$ insulin, $1 \mu \mathrm{M}$ dexamethasone, $0.5 \mathrm{mM}$ 3-isobutyl-1-methylxanthine, and $300 \mu \mathrm{M}$ oleate (dissolved in DMSO) (all from Sigma-Aldrich, St. Louis, MO, USA).

\section{Cell transfection}

Chicken intramuscular preadipocytes were seeded at a density of $1 \times 10^{5}$ cells $/ \mathrm{mL}$ in 6 -well plates. Upon reaching 60-70\% confluence, the cells were transfected with a gga-miR-140-5p mimic (50 $\mathrm{nM})$ or a negative control (50 nM) using $4 \mu \mathrm{L}$ of Lipofectamine 2000, and the medium was replaced $6 \mathrm{~h}$ later. After 24 $\mathrm{h}$, the cell medium was replaced with differentiation medium.

\section{Quantitative real-time PCR analysis}

The relative expression levels of ten randomly selected DEGs and DE miRNAs were validated using quantitative real-time reverse transcription PCR (qRT-PCR). The specific and efficient primers for reverse transcription of stem-loop miRNAs in this study were purchased from GenePharma Co., Ltd. (Shanghai, China) [26]. The final concentration of each primer was $10 \mu \mathrm{mol} / \mu \mathrm{L}$. The primers used are described in Table 3. Total RNA (tissues and cells) was extracted using TRIzol reagent, and then was reverse transcribed using the PrimeScript RT reagent Kit with gDNA Eraser (TaKaRa) following the manufacturer's instructions. qRT-PCR was performed in triplicate using the SYBR Premix Ex Taq II kit (TaKaRa) on a LightCycler 96 instrument (Roche, Indianapolis, IN, USA). Chicken U6 RNA and GAPDH were chosen as endogenous internal controls. The $2^{-\Delta \Delta C t}$ method was used to determine the relative mRNA and miRNA abundance [27].

\section{Oil Red $O$ staining and quantification}

Frozen tissues were sectioned using a Leica Kryostat (CM3050S, Leica Microsystems GmbH, Wetzlar, Germany), fixed 30 min with 10\% paraformaldehyde, and incubated 15 min at room temperature with Oil Red 0 , then visualized by microscopy. For quantification of tissue Oil Red 0 staining, each slice within each group was randomly selected at least 3 times within the field of vision $(200 \times)$ to obtain images. The same red color as the uniform standard for evaluating the lipid droplets in all the images was applied with ImagePro Plus 6.0 software (Media Cybernetics, Inc., Rockville, MD, USA). Fat droplets were quantified using the following equation: percentage fat droplets $=$ (red lipid droplet area/total tissue area $\times 100)(n=6)$. For cellular Oil Red 0 staining, cells were washed with PBS three times and fixed with $10 \%$ formaldehyde for 30 min. The fixed cells were washed with PBS three times and incubated with $1 \%$ filtered Oil Red 0 solution for $40 \mathrm{~min}$, and then observed under a phase-contrast microscope to check for positive cells appearing red. Subsequently, Oil Red 0 was eluted from the stained cells with $100 \%$ isopropanol (v/v) and quantified by microplate reader (Thermo Fisher Scientific) at $500 \mathrm{~nm}$.

\section{Statistical analyses}

All experiments were carried out at least three times. Data are presented as the mean \pm standard error of the mean based on at least three replicates for each treatment. Graphics were drawn using the "ggplot2" package in R (version 3.2.2) and GraphPad Prism 7 software (San Diego, CA, USA). Data were analyzed using SPSS 19.0 software (IBM, Chicago, IL, USA) and evaluated for differences between groups by unpaired Student's $t$ test. ${ }^{*} P<0.05$; ${ }^{* *} P<0.01$. 


\section{Cellular Physiology Cell Physiol Biochem 2018;46:2421-2433 and Biochemistry Publis.1159/000489649 $\begin{aligned} & \text { DOI: 10.11 } 2018 \text { The Author(s). Published by S. Karger AG, Basel } \\ & \text { www.karger.com/cpb }\end{aligned}$ \\ Zhang et al.: The Effection of miRNA on Chicken Intramuscular Fat Depositon}

\section{Results}

Differences in IMF of breast muscle tissues between juvenile and late-laying-period Gushi hens

Given the tremendous differences in meat quality between juvenile and late-layingperiod Gushi hens and to visualize the differences in IMF deposition [18], Oil Red 0 staining of breast muscle samples was performed in the present study. Our results showed that the density and size of lipid droplets in the G55W group were greater than those of the G20W group (Fig. 1), which is consistent with previous results obtained by the Soxhlet extraction method [18].

\section{Overview of small RNA deep sequencing data}

A total of 40, 716, 792 and 54, 602, 198 raw reads in the range of 18-30 nt were obtained from the G20W and G55W libraries, respectively. After removing contaminant reads, we obtained 39, 435, 411 (G20W) and 53, 371, 476 (G55W) clean reads that were used for subsequent analyses (Table 1). A total of 502 miRNAs were obtained from all samples, of which 320 were known miRNAs and 182 were newly predicted miRNAs. Eighty percent of known and newly predicted miRNAs were 21-23 nt in length in the G20W and G55W libraries (Fig. 2A). The majority of the miRNA reads were 22 and $23 \mathrm{nt}$ in length in both groups (Fig. 2B). Compared with the G20W group, the expression levels of known and novel miRNAs were lower in the G55W group (Fig. 2C).

\section{Identification of differentially expressed miRNAs}

To identify the DE miRNAs that may play important regulatory roles in chicken meat quality, especially IMF content, we compared the expression levels of miRNAs between the juvenile and late-laying-period groups. Pearson correlation analysis showed that there was a high correlation between the samples in the group, indicating that the sampling was reasonable (Fig. 3A). A total of 104 miRNAs exhibited significantly different expression among the G20W and G55W groups (Fig. 3B). Among the 104 DE miRNAs, 100 were downregulated in the G55W group and 4 were upregulated in the G20W group. The majority of DE miRNAs were downregulated in the G55W group, including miR-9-3p, miR-206, miR-222b-5p, miR-222b-3p, miR-140-5p, and miR-223 (Fig. 3C).

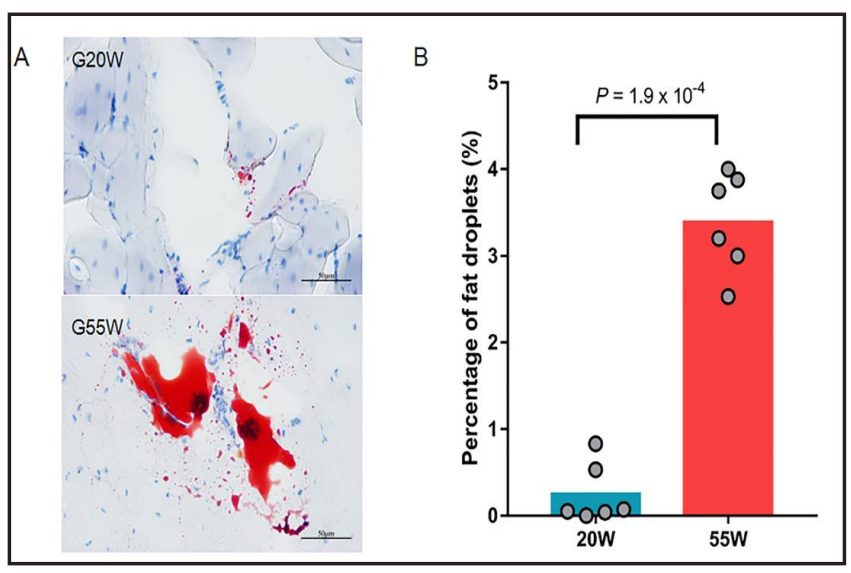

Fig. 1. Oil Red 0 staining for neutral lipids in breast muscle of juvenile and late laying-period Gushi hens (400X). Breast muscle samples were paraformaldehyde fixed and incubated with Oil Red 0 and visualized by microscopy. (A) Lipids in G20W and G55W group. (B) The quantification of Oil red 0 staining.

Table 1. Descriptive summary of non-coding RNAs reads. ${ }^{a} 20 \mathrm{~W}$, breast muscle samples from 20 -week-old

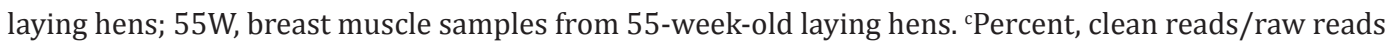

\begin{tabular}{lcccccc}
\hline Samples $^{\mathrm{a}}$ & Samples ID $^{\mathrm{b}}$ & Total_reads & Clean_reads & Percent(\%)c & Mapped_Reads & Q30(\%) \\
\hline $20 \mathrm{~W}-1$ & S01 & 13142570 & 12808455 & 97.50 & 8008281 & 98.90 \\
$20 \mathrm{~W}-2$ & S02 & 13196925 & 12696714 & 96.21 & 7194320 & 98.90 \\
$20 \mathrm{~W}-3$ & S03 & 14377160 & 13930242 & 96.89 & 8989611 & 98.97 \\
$55 \mathrm{~W}-1$ & S04 & 15704916 & 15376238 & 97.91 & 10270028 & 99.28 \\
$55 \mathrm{~W}-2$ & S05 & 16945145 & 16455958 & 97.11 & 10986997 & 99.28 \\
$55 \mathrm{~W}-3$ & S06 & 21952088 & 21539280 & 98.12 & 15492928 & 99.39 \\
\hline
\end{tabular}


Target gene prediction and functional enrichment analysis

To characterize the regulatory roles of miRNAs in chicken meat quality, DE miRNA target prediction, GO enrichment, and KEGG pathway analyses were performed in this study. A total of 9, 601 target genes for the $104 \mathrm{DE}$ miRNAs were identified. All of the target genes were mainly enriched in very-long-chain fatty acid metabolic processes, fatty acid beta-oxidation, fatty-acyl-CoA binding, ubiquitin-conjugating enzyme activity, and extracellular exosome biological processes (Fig. 4A). The significantly enriched pathways included valine, leucine, and isoleucine degradation, protein processing in the endoplasmic reticulum, insulin signaling, Notch signaling, fatty acid degradation, and extracellular matrix-receptor interactions (Fig. 4B).

\section{Integrated analysis of differentially expressed miRNAs and mRNA}

To better understand the regulatory roles of miRNAs in chicken meat quality, we integrated the target genes of the DE miRNAs and DEGs obtained from the chicken breast

Fig. 2. Summary of miRNA-seq data. (A) Length distribution of miRNA sequences in different categories (Total, Known and Novel). (B) Size distribution of sequenced small RNA reads. (C) Expression levels of miRNAs between juvenile and late laying-period Gushi hens in different categories (All, Known and Novel).

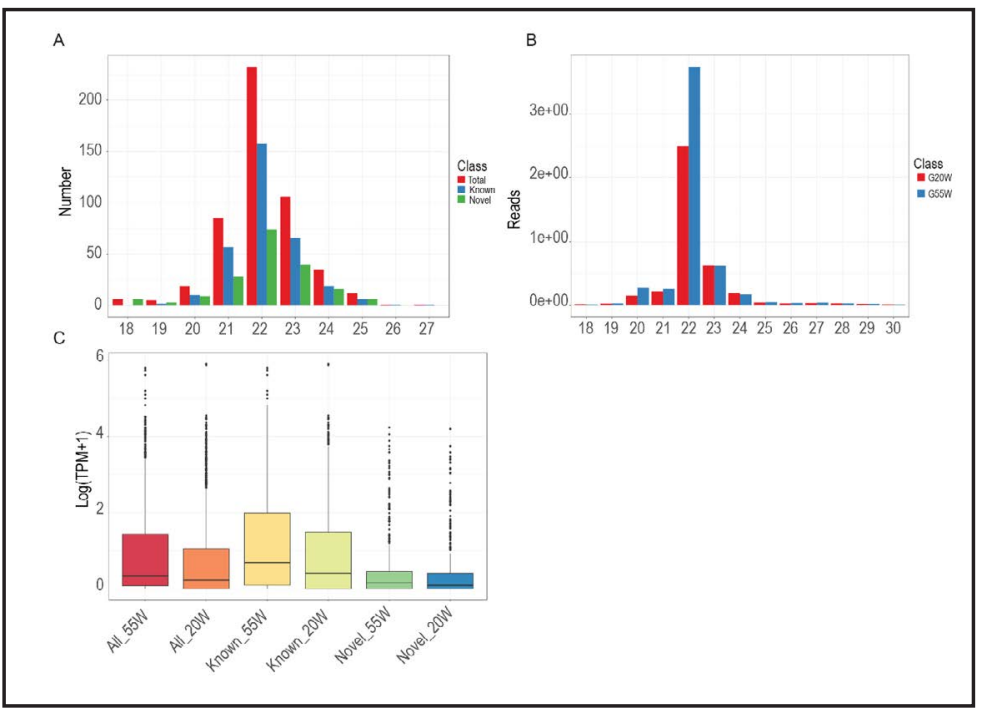

Fig. 3. Overview of small RNA Deep Sequencing Data. (A) Correlation analysis between samples. Venn plot (B) and volcano plot (C) of DE miRNAs between two groups. The green and red triangles indicate downregulated and up-regulated miRNAs in G55W group, respectively. 
muscle transcriptome data (manuscript in press). Furthermore, we found that those target genes of the DE miRNAs were mainly enriched in ubiquitinmediated proteolysis, peroxisome proliferator-activated receptor (PPAR) signaling, glycerophospholipid metabolism, fatty acid metabolism, fatty acid elongation, fatty acid degradation, and biosynthesis of unsaturated fatty acids (Fig. 5). To further understand the function of the intersecting genes in breast muscle quality, we visualized the integrated miRNA-mRNA networking among the DE miRNAs and their target genes significantly enriched in the above-mentioned pathways (Fig. 6). Our results showed that a large number of DEGs related to lipid metabolism, including lysophosphatidylcholine acyltransferase 2, hydroxyacyl-CoA dehydrogenase, alpha subunit (HADHA), hydroxyacyl-CoA dehydrogenase, beta subunit (HADHB), ELOVL fatty acid elongase 2, 5, and 6 (ELOVL2, ELOVL5, ELOVL6), 1-acylglycerol-3-phosphate $O$ acyltransferase 3 ( $A G$ PAT3), and perilipin 2 (Table 2) were potentially targeted by the significantly upregulated miRNAs, includ-

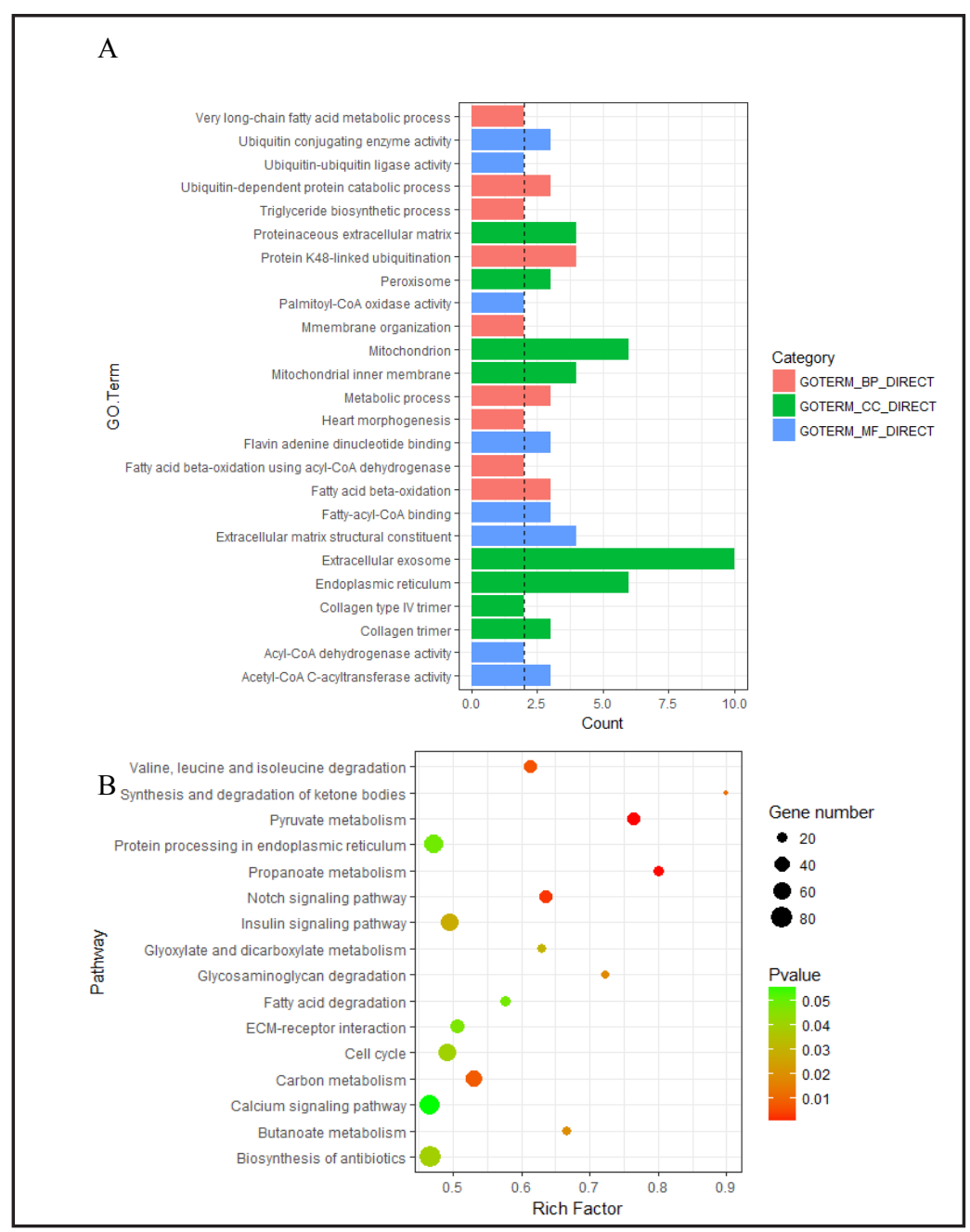

Fig. 4. Functional enrichment analysis of DE miRNAs. (A) GO (Gene Ontology) categories enrichment analysis of target genes. (B) KEGG pathways enrichment analysis of target genes.

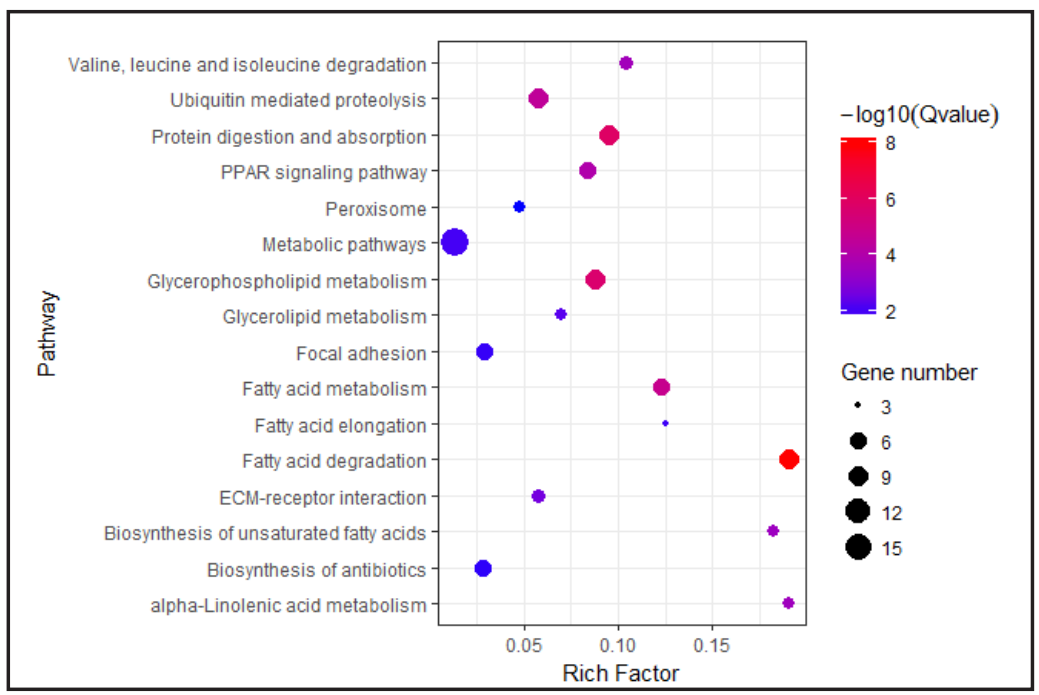

Fig. 5. KEGG pathways of DEGs targeted by DE miRNAs. 
ing miR-218-5p, miR124a-3p, miR-181b-5p, and miR-130b-3p (Fig. 6).

qRT-PCR validation
of the sequencing
data

To validate the RNASeq data, we selected 10 DEGs (7upregulated and 3 downregulated genes) and $8 \mathrm{DE}$ miRNAs and determined the expression of these RNAs by qRT-PCR. Our results showed that the expression of the selected mRNAs and miRNAs was consistent with the RNA-Seq results (Fig. 7).

\section{Effect of gga-miR- 140-5p on the differentiation of intramuscular adipocytes}

To further explore the biological significance of candidate DE miRNAs, we focused on gga-miR140-5p, which was downregulated in the breast muscle of late-laying-period hens. We noticed that the mature miR-140-5p sequence was highly conserved in various species including cattle, pig, zebra finch, human, and mouse (Fig. 8A). To determine the tissue expression patterns of gga-miR140-5p, 10 tissues of Gushi hens were analyzed using qRT-PCR. The results showed that gga-miR-140-5p was highly expressed in abdominal fat, ovary, and duodenum (Fig. 8B). To investigate the expression patterns of gga-miR-140-5p during chicken intramuscular adipocyte differentiation, we isolated the intramuscular preadipocytes. After 10 days of induction, intramuscular adipocytes were fully differentiated and filled with large lipid droplets (Fig. 8C). Total RNA was then extracted from adipogenic cells at 0, 2, 4, 6, 8, and 10 days of differentiation to detect adipocyte markers and the gga-miR-140-5p expression pattern. Our results suggested that the mRNA levels of the adipocyte markers PPAR $\gamma(P P A R G)$ and fatty acid binding protein 4 (FABP4) increased with adipocyte differentiation (Fig. 8D). Furthermore, the mRNA levels of gga-miR-140-5p and $R X R G$ were detected by qRT-PCR. Our results showed that the expression level of gga-miR-140-5p significantly increased (Fig. 9A), while that of RXRG significantly decreased during intramuscular adipocyte adipogenesis (Fig. 9B). Taken together, these data suggested that gga-miR-140-5p might be associated with intramuscular adipocyte adipogenesis. 
gga-miR-140-5p promotes intramuscular adipocyte differentiation by targeting RXRG

To explore the regulatory role of gga-miR-140-5p in intramuscular adipocyte differentiation, gga-miR-140$5 p$ was overexpressed by using miRNA mimic. Compared with the negative control, the expression level of gga-miR$140-5 p$ significantly increased in chicken adipocytes after transfection with the ggamiR-140-5p mimic (Fig. 10A). Compared with the control group, the level of RXRG, PPARG, and FABP4 significantly increased in the gga-miR-140-5poverexpressing group (Fig. 10B). Oil Red 0 staining suggested that the number of intracellular lipid droplets was increased in the gga-miR-140-5p-overexpressing group (Fig. 10C and 10E). To verify the direct binding site between miR-140-5p and $R X R G$, a $3^{\prime}$ UTR fragment with a seed region binding site was inserted into the $3^{\prime}$ UTR of the Renilla luciferase (Rluc) gene of the psiCHECK-2 vector (Fig. 10D). Luciferase assay revealed that gga-miR$140-5 p$ significantly reduced the Rluc activity of the wild-type RXRG reporter vector, whereas point mutations of target sites bound by the seed region of ggamiR-140-5p in the $3^{\prime}$ UTR of RXRG did not disrupt luciferase activity (Fig. 10F). Taken together, these results suggest that gga-miR-140-5p promotes adipocyte differentiation by downregulating $R X R G$.

All the Illumina miRNA-seq data sets supporting the results of this article have been submitted to the National Center for Biotechnology Information (NCBI) Sequence Read Archive (SRA) under accession number SRR6347620, SRR6347621,SRR6347622,SRR6347623 SRR6347624, SRR6347625.

\section{Discussion}

Muscle is mainly composed of muscle fibers, fat, and connective tissue. Collagen is the main component of connective tissue, which is closely related with tenderness. Due to the different ages of poultry and the proportion of connective tissue in each part of muscle, the tenderness of meat products can be significantly different. Meat quality is affected by many factors, among which IMF content contributes to the juiciness and tenderness of the meat. IMF is mainly deposited in
Table 3. List of cloning and qPCR primers used in the present study

\begin{tabular}{|c|c|c|c|c|c|}
\hline Accession No. & $\begin{array}{c}\text { Gene } \\
\text { symbol }\end{array}$ & Primer sequence & $\begin{array}{l}\text { Anneaning } \\
\text { temperature }\end{array}$ & $\begin{array}{c}\text { Product } \\
\text { size }\end{array}$ & Purpose \\
\hline NM_001001753.1 & CYP7A1 & $\begin{array}{l}\text { F: AGGACTTTCGTCTGGCTCT } \\
\text { R: CTCCGCATCGGGTATTT }\end{array}$ & $60^{\circ} \mathrm{C}$ & 185 bp & QPCR \\
\hline NM_001031420.1 & PLIN2 & $\begin{array}{l}\text { F: TCTGTCTTCAAAGTTCCGTG } \\
\text { R: TCTTTCTGGCATACTCGATCA }\end{array}$ & $60^{\circ} \mathrm{C}$ & $131 \mathrm{bp}$ & QPCR \\
\hline XM_004934600.2 & AGPAT3 & $\begin{array}{l}\text { F: TGGCCAGCAGTTTAAACCAC } \\
\text { R: TCCCAGGAATGCAAGGATAAGG }\end{array}$ & $60^{\circ} \mathrm{C}$ & $145 \mathrm{bp}$ & QPCR \\
\hline NM_001031145.1 & AGPAT9 & $\begin{array}{l}\text { F: TGCGATCATCTTGACCAACG } \\
\text { R: AATTTCCGAGCGCTCAAACC }\end{array}$ & $60^{\circ} \mathrm{C}$ & $127 \mathrm{bp}$ & QPCR \\
\hline NM_001031288.1 & DHCR24 & $\begin{array}{l}\text { F: ACCGCATACCTGAATCCTATGG } \\
\text { R: TGCAGGTGTGTTGGAAAAGC }\end{array}$ & $60^{\circ} \mathrm{C}$ & $135 \mathrm{bp}$ & QPCR \\
\hline NM_001030568.2 & LPCAT2 & $\begin{array}{l}\text { F: GGAGGTTGAGTTTCTGCCTGT } \\
\text { R: TGACTGGCACCTTCAAAGCA }\end{array}$ & $60^{\circ} \mathrm{C}$ & $113 \mathrm{bp}$ & QPCR \\
\hline NM_205107.1 & COL6A1 & $\begin{array}{l}\text { F::GTGTTGGATATTTTTCAAAGTGT } \\
\text { R: CTATTCTTCAACTCAACCCTACC }\end{array}$ & $60^{\circ} \mathrm{C}$ & $142 \mathrm{bp}$ & QPCR \\
\hline NM_001293134.1 & COLBA1 & $\begin{array}{l}\text { F: TGCCCGTGAAGTTTGACAAG } \\
\text { R: TTGAACAAAGCCACCCAGAC }\end{array}$ & $60^{\circ} \mathrm{C}$ & 128 bp & QPCR \\
\hline NM_204145.2 & $\mathrm{ABCA} 1$ & $\begin{array}{l}\text { F: TCCTCTGGCTTAGACTTGA } \\
\text { R: CTCGTAGTTGTATTCGGTAA }\end{array}$ & $60^{\circ} \mathrm{C}$ & $164 \mathrm{bp}$ & QPCR \\
\hline NM_001039602.1 & SLC27A1 & $\begin{array}{l}\text { F: TACGGAGCCACCGAGTGCAACT } \\
\text { R: CGCACAGCCCTCTGGAATCACG }\end{array}$ & $60^{\circ} \mathrm{C}$ & $160 \mathrm{bp}$ & QPCR \\
\hline NM_205294.1 & RXRG & $\begin{array}{l}\text { F: TACAGGGTCATCGCATCCTC } \\
\text { R: CCCCATAGTGCTTCCCTGAA }\end{array}$ & $60^{\circ} \mathrm{C}$ & $150 \mathrm{bp}$ & QPCR \\
\hline NM_001001460.1 & PPARG & $\begin{array}{l}\text { F: GTGCAATCAAAATGGAGCC } \\
\text { R: CTTACAACCTTCACATGCAT }\end{array}$ & $60^{\circ} \mathrm{C}$ & $170 \mathrm{bp}$ & QPCR \\
\hline NM_204290.1 & FABP4 & $\begin{array}{l}\text { F: ATGTGCGACCAGTTTGT } \\
\text { R: TCACCATTGATGCTGATAG }\end{array}$ & $60^{\circ} \mathrm{C}$ & 143 bp & QPCR \\
\hline NM_204328.1 & Fox01 & $\begin{array}{l}\text { F:ATCCGTCACAACTTGTCCCT } \\
\text { R:ACCATTTCCCTCCTGACCAG }\end{array}$ & $60^{\circ} \mathrm{C}$ & $219 \mathrm{bp}$ & QPCR \\
\hline NM_204305.1 & GAPDH & $\begin{array}{l}\text { F: AGAACATCATCCCAGGGT } \\
\text { R: AGCCTTCACTACCCTCTTG }\end{array}$ & $60^{\circ} \mathrm{C}$ & $184 \mathrm{bp}$ & QPCR \\
\hline NC_006095.4 & $\begin{array}{l}\text { RXRG- } \\
\text { 3'UTR- } \\
\text { WT }\end{array}$ & $\begin{array}{l}\text { F: CCTCTTTGGTGGGATTGTTG } \\
\text { R: ACAGCAAAGCAGGACGATG }\end{array}$ & $61^{\circ} \mathrm{C}$ & $540 \mathrm{bp}$ & Colning \\
\hline NC_006095.4 & $\begin{array}{l}\text { RXRG- } \\
\text { 3'UTR- } \\
\text { Mut }\end{array}$ & $\begin{array}{l}\text { F:TGCGGACCCTCTGCCCAGCTACACCTCCCTGTACAGATAA } \\
\text { R:TTATCTGTACAGGGAGGTGTAGCTGGGCAGAGGGTCCGCA }\end{array}$ & $61^{\circ} \mathrm{C}$ & 533 bp & Colning \\
\hline
\end{tabular}

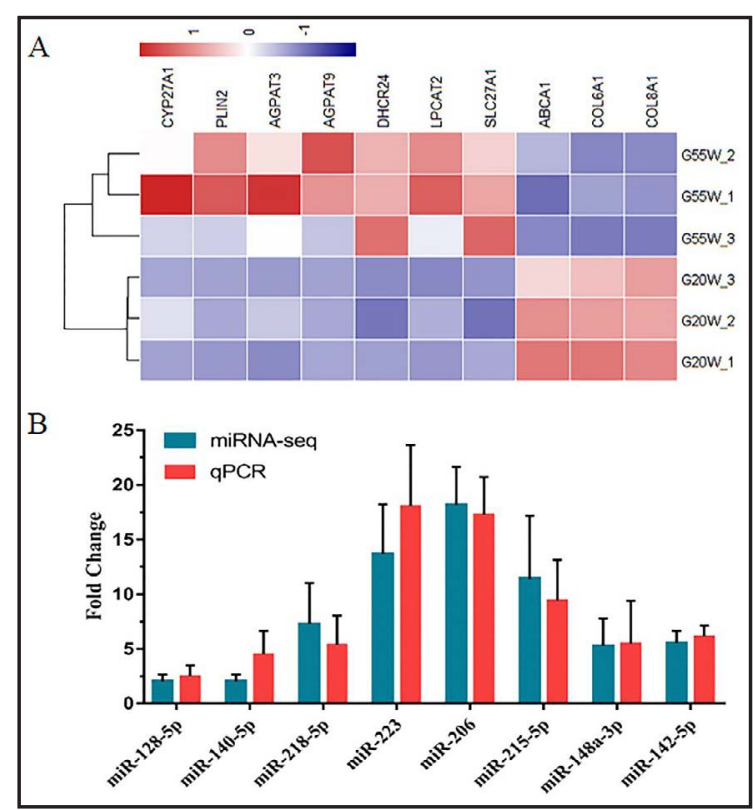

Fig. 7. Validation of DEGs and DE miRNAs by qPCR. 
Fig. 8. Expression profile of gga-miR140-5p in different tissues and the model building of chicken intramuscular preadipocyte differentiation. (A) Mature miR-140$5 p$ sequence was conserved among species. (B) Tissue expression profile of gga-miR-140-5p in Gushi hens. (C)

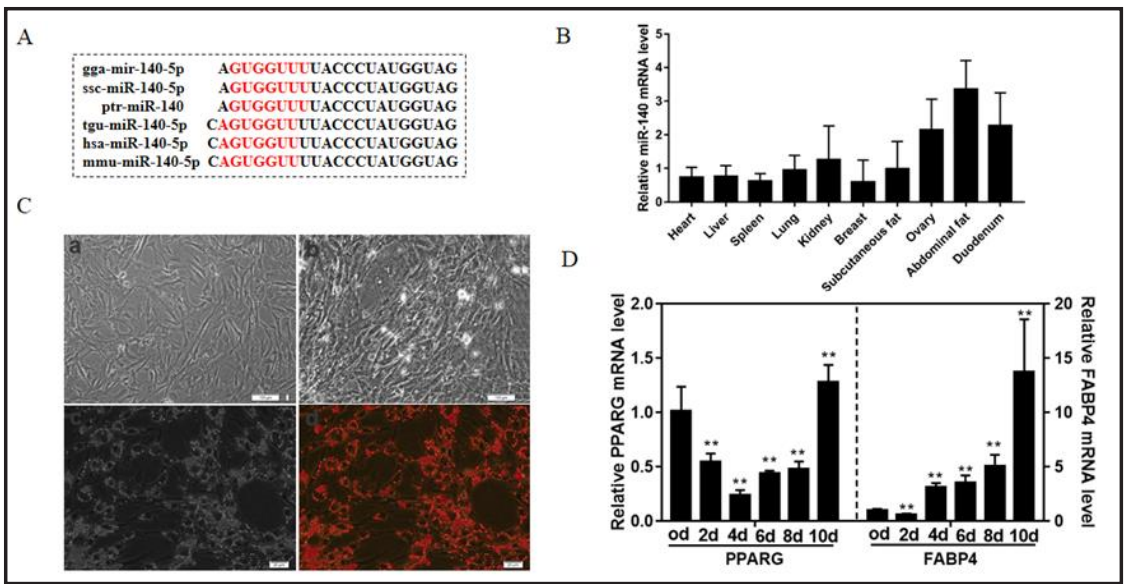

Photomicrographs showing cell morpghology change during chicken intramuscular preadipocyte differentiation. (a) Chicken intramuscular preadipocyte before IBMX-DEX-Insulin-Oleate (DMIO) induction; (b)\& (c) chicken intramuscular preadipocyte 4 and 6 days after DMIO induction; (d) chicken intramuscular preadipocyte 6 days after DMIO induction staining with Oil Red 0). (D) The relative mRNA expression level of adipocyte maker genes PPARG and FABP4 during chicken intramuscular preadipocyte differentiation $(\mathrm{n}=$ $\left.3,{ }^{* *} \mathrm{p}<0.01\right)$

Fig. 9. Expression of ggamiR-140-5p (A) and RXRG (B) during chicken intramuscular preadipocyte differentiation $\left(\mathrm{n}=3,{ }^{* *} \mathrm{p}<0.01\right)$.

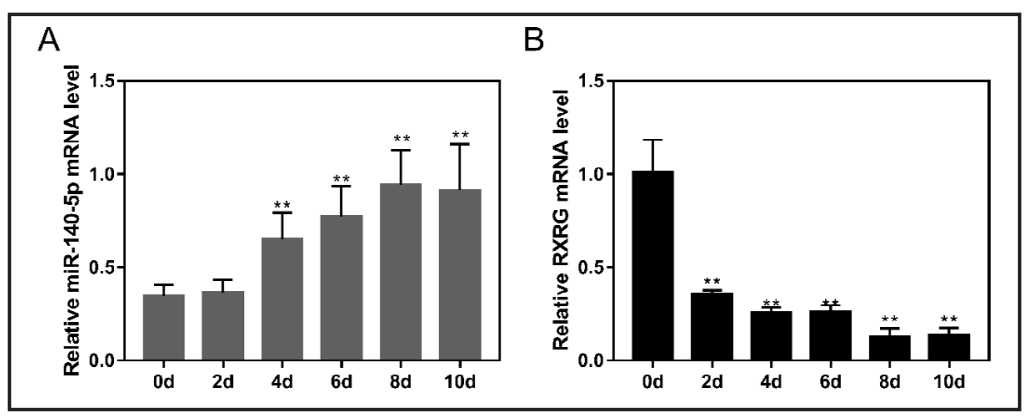

Fig. 10. gga-miR-140-5p promotes chicken instramuscular preadipocyte differentiation by targeting the 3'UTR of RXRG. (A) Overexpression of gga-miR-140-5p. (B) mRNA levels of PPARG, FABP4, FOX01 and RXRG were analyzed by qRT-PCR ( $n=3$ ). (C) Oil Red O staining of intramuscular adipocytes after transfection with ggamiR-140-5p mimic. (D) Target site of gga-miR-140-5p within chicken RXRG mRNA 3'UTR and the mutation design of its 3'UTR; (E) Differ-
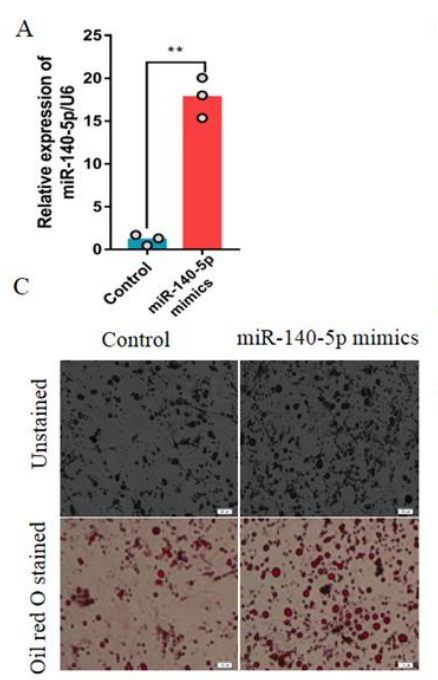

B

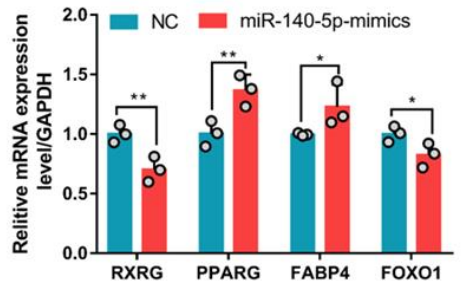

D RXRG $3^{\prime}$ UTR mutation, ACCCUCUGCCCAGCUTCGTCGAC. RXRG $3^{\prime}$ UTR S'. ACCCUCUGCCCAGCUAACCACAC. gep-mir-140.5p $\quad{ }^{3}$ GAUGGUAUCCCAUUUUGGGUGA

E

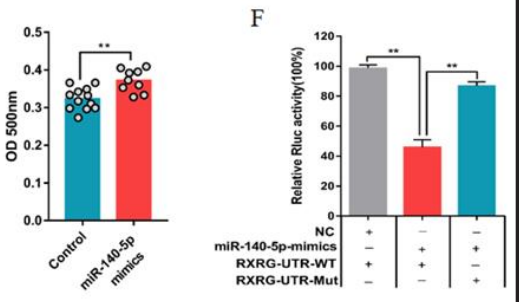
entiation of adipocytes was identified by Oil Red 0 staining ( $\mathrm{n}=9$ ). (F) gga-miR-140-5p mimics was transfected into DF1 cells along with RXRG-3'UTR-WT or RXRG-3'UTR-Mut. (**p<0.01). 


\section{Cellular Physiology Cell Physiol Biochem 2018;46:2421-2433 \\ \begin{tabular}{l|l} 
and Biochemistry Published onlIne: May 09, 2018 & $\begin{array}{l}\text { DO } 2018 \text { The Author(s). Published by S. Karger AG, Basel } \\
\text { www.karger.com/cpb }\end{array}$ \\
\hline
\end{tabular}}

Zhang et al.: The Effection of miRNA on Chicken Intramuscular Fat Depositon

the muscle mass with fat, distributed in the myometrium, myofibrils, and endometrium, as well as in the form of lipid droplets in the muscle cytoplasm, which includes intracellular phospholipid, triglyceride, and cholesterol.

In our previous study, we found that the late-laying-period hens exhibited a higher serum lipid level and water holding capacity, with a lower muscle tenderness than the juvenile hens [18]. Furthermore, a large number of genes related to meat quality including AGPAT3, AGPAT9, HADHA, HADHB, LPL, ACAA2, and SLC27A1 were found differentially expressed in the two physiological stages. However, the molecular mechanisms and roles of miRNAs affecting IMF are still unclear.

Our previous studies have shown that a large number of miRNAs may target genes related to chicken hepatic lipid metabolism between pre- and peak-laying stages [28]. The adipocytes of poultry have a weak lipid synthesis capability compared with mammals, and the lipid synthesis is predominantly in the liver [29]. Lipids synthesized by chicken liver are mainly transported to muscle and adipose tissues. In the current study, we investigated the expression profile of breast muscle miRNAs related to the mechanism underlying differences in meat quality in chickens at two different physiological stages (20 and 55 weeks). In total, 320 known and 182 potential novel miRNAs were identified in this study. Among them, One hundred miRNAs were downregulated in late-laying-period hens, which suggested that those miRNAs may play important roles in gene upregulation. Previous studies demonstrated that miR-19b-3p [30], miR-425-5p [16], miR-27a [31], miR-146-5p [32], and miR-223 [33] influenced the differentiation of preadipocytes. We found that most of those miRNAs were DE miRNAs in the present study. Interestingly, we found that the majority of the miRNAs target genes were associated with glycerophospholipid metabolism, fatty acid synthesis, fatty acid elongation and degradation, and ubiquitin-mediated proteolysis.

To demonstrate the applicability of the approach used in this study, we focused on one of the DE miRNAs, gga-miR-140-5p, which was originally found to be specifically expressed in the cartilage of zebrafish and mouse embryos [34, 35]. Yan et al [36]. found that gga-miR-140-5p inhibits hepatocellular carcinoma by directly targeting Pin1 to block multiple cancer-driving pathways. Zhang et al [37]. discovered that miR-140-5p regulates adipocyte differentiation by targeting TGFBR1. To our knowledge, no previous studies have associated gga-miR-140$5 \mathrm{p}$ with chicken IMF deposition. In this study, we aimed to investigate the physiological role of gga-miR-140-5p associated with adipogenesis in chicken intramuscular preadipocytes. Our results showed that gga-miR-140-5p level was increased during intramuscular preadipocyte differentiation, indicating that gga-miR-140-5p might regulate intramuscular preadipocytes. Furthermore, gga-miR-140-5p was found to potentially combine with the $3^{\prime} \mathrm{UTR}$ of $R X R G$. RXRG, which belongs to the steroid/thyroid hormone receptor superfamily of ligand-activated transcription factors, usually binds to the promoter region together with $P P A R G$ involved in gene transcription [38]. In our study, overexpression of gga-miR-140-5p in chicken intramuscular preadipocytes increased the expression of PPARG and FABP4, while it decreased that of $R X R G$ and FOXO1. As expected, Oil Red $O$ staining showed an increased lipid accumulation in the gga-miR-140-5p-overexpressing group. Furthermore, luciferase reporter assay showed that gga-miR-140-5p significantly downregulated the luciferase activity of the wild-type $R X R G$ reporter in DF1 cells compared with control and negative (point mutations of target sites) groups. Taken together, our findings showed that ggamiR-140-5p is a novel promoter of chicken intramuscular adipogenesis possibly through targeting $R X R G$.

In conclusion, our study identified miRNAs that are associated with chicken meat quality between pre-laying and late-laying-period hens. We constructed a miRNA-mRNA regulatory network that may affect chicken breast meat quality. Moreover, our findings showed that gga-miR-140-5p could promote adipogenic differentiation by negatively regulating $R X R G$. Our findings may serve as a fundamental resource for studies about improving poultry meat quality and also contribute to a more thorough understanding of chicken IMF deposition. 


\section{Cellular Physiology Cell Physiol Biochem 2018;46:2421-2433 and Biochemistry Published onIIne: May 09, $2018 \quad \begin{aligned} & \text { DOI: 10.1159/000489649 } \\ & \begin{array}{l}\text { C } 2018 \text { The Author(s). Published by S. Karger AG, Basel } \\ \text { www.karger.com/cpb }\end{array}\end{aligned}$

\section{Acknowledgements}

This work was supported by China Agriculture Research System (CARS-40-K04), Program for Innovation Research Team of Ministry of Education (IRT16R23), Key Science and Technology Research Project of Henan Province (151100110800), National Natural Science Foundation of China (31572356), Research on the Foundation and Frontier Technology of Henan Science and Technology Department (162300410162).

Gui-Rong Sun and Xiang-Tao Kang and conceived and designed the experiments. Meng Zhang, Dong-Hua Li and Fang Li contributed to the slaughter experiment, cell experiment, data analysis, and wrote the manuscript. Jun-Wei Sun and contributed to the sample preparation and qRT-PCR. Rui-Rui Jiang, Zhuan-Jian Li, Rui-Li Han and Xiao-Jun Liu contributed to the experimental design and helped to draft the manuscript. All authors reviewed and approved the final manuscript.

\section{Disclosure Statement}

The authors declare to have no conflict of interests.

\section{References}

1 Cameron ND, Nute GR, Brown S, Enser M, Wood J: Meat quality of Large White pig genotypes selected for components of efficient lean growth rate. Anim Sci 1999;68:115-127.

-2 Fischer K: Drip loss in pork: influencing factors and relation to further meat quality traits. J Anim Breed Genet 2015;124:12-18.

3 Dokmanovic M, Ivanovic J, Janjic J, Boskovic M, Laudanovic M, Pantic S, Baltic M: Effect of lairage time, behaviour and gender on stress and meat quality parameters in pigs. Anim Sci J 2016;88:500-506.

4 Tellam RL, Cockett NE, Vuocolo T, Bidwell CA: Genes Contributing to Genetic Variation of Muscling in Sheep. Front Genet 2012;3:164.

5 Fanatico AC, Pillai PB, Emmert JL, Owens CM: Meat Quality of Slow- and Fast-Growing Chicken Genotypes Fed Low-Nutrient or Standard Diets and Raised Indoors or with Outdoor Access. Poult Sci 2007;86:22452255.

-6 Hou X, Yang Y, Zhu S, Hua C, Zhou R, Mu Y, Tang Z, Li K: Comparison of skeletal muscle miRNA and mRNA profiles among three pig breeds. Mol Genet Genomics 2016;291:559-573.

7 Wood JD, Richardson RI, Nute GR, Fisher AV, Campo MM, Kasapidou E, Sheard PR, Enser M: Effects of fatty acids on meat quality: a review. Meat Sci 2004;66:21.

-8 Bartel DP: MicroRNA Target Recognition and Regulatory Functions. Cell 2009;136:215.

-9 Johnson CD, Esquelakerscher A, Stefani G, Byrom M, Kelnar K, Ovcharenko D, Wilson M, Wang X, Shelton J, Shingara J: The let-7 microRNA represses cell proliferation pathways in human cells. Cancer Res 2007;67:7713-7722.

10 Chen JF, Mandel EM, Thomson JM, Wu Q Callis TE, Hammond SM, Conlon FL, Wang DZ: The role of microRNA-1 and microRNA-133 in skeletal muscle proliferation and differentiation. Nat Genet 2006;38:228-223.

-11 Esau C, Kang X, Peralta E, Hanson E, Marcusson EG, Ravichandran LV, Sun Y, Koo S, Perera RJ, Jain R: MicroRNA-143 regulates adipocyte differentiation. J Biol Chem 2004;279:52361.

12 Cheng AM, Byrom MW, Shelton J, Ford LP: Antisense inhibition of human miRNAs and indications for an involvement of miRNA in cell growth and apoptosis. Nucleic Acids Res 2005;33:1290-1297.

13 Alvarez-Garcia I, Miska EA: MicroRNA functions in animal development and human disease. Development 2005;132:4653.

14 Kim HK, Lee YS, Sivaprasad U, Malhotra A, Dutta A: Muscle-specific microRNA miR-206 promotes muscle differentiation. J Cell Biol 2006;174:677-687.

15 Lin Q, Gao Z, Alarcon RM, Ye J, Yun Z: A Role of miR-27 in the Regulation of Adipogenesis. Febs J 2010;276:2348-2358. 


\section{Cellular Physiology Cell Physiol Biochem 2018;46:2421-2433 \begin{tabular}{l|l} 
DOI: 10.1159/000489649 & $\begin{array}{l}\text { O 2018 The Author(s). Published by S. Karger AG, Basel } \\
\text { www.karger.com/cpb }\end{array}$
\end{tabular} \\ Zhang et al.: The Effection of miRNA on Chicken Intramuscular Fat Depositon}

16 Chen FF, Xiong Y, Peng Y, Gao Y, Qin J, Chu GY, Pang WJ, Yang GS: miR-425-5p Inhibits Differentiation and Proliferation in Porcine Intramuscular Preadipocytes. Int J Mol Sci 2017;18:2101.

17 Shen L, Du J, Xia Y, Tan Z, Fu Y, Yang Q, Li X, Tang G, Jiang Y, Wang J: Genome-wide landscape of DNA methylomes and their relationship with mRNA and miRNA transcriptomes in oxidative and glycolytic skeletal muscles. Sci Rep 2016;6:32186.

18 Zhang M, Yan FB, Li F, Jiang KR, Li DH, Han RL, Li ZJ, Jiang RR, Liu XJ, Kang XT, Sun GR: Genome-wide DNA methylation profiles reveal novel candidate genes associated with meat quality at different age stages in hens. Sci Rep 2017;7:45564.

19 Griffithsjones S, Saini HK, Van DS, Enright AJ: miRBase: tools for microRNA genomics. Nucleic Acids Res 2008;36:154-158.

20 Friedländer MR, Mackowiak SD, Li N, Chen W, Rajewsky N: miRDeep2 accurately identifies known and hundreds of novel microRNA genes in seven animal clades. Nucleic Acids Res 2012;40:37-52.

21 Yang J, Zhang F, Li J, Chen JP, Zhang HM: Integrative Analysis of the microRNAome and Transcriptome Illuminates the Response of Susceptible Rice Plants to Rice Stripe Virus. Plos One 2016;11.

22 Wang L, Feng Z, Wang X, Wang X, Zhang X: DEGseq: an R package for identifying differentially expressed genes from RNA-seq data. Bioinformatics 2010;26:136-138.

23 Jr DG, Sherman BT, Hosack DA, Yang J, Gao W, Lane HC, Lempicki RA: DAVID: Database for Annotation, Visualization, and Integrated Discovery. Genome Biol 2003;4:P3.

24 Smoot ME, Ono K, Ruscheinski J, Wang PL, Ideker T: Cytoscape 2.8. Bioinformatics 2011;27:

25 Zhang T, Zhang X, Han K, Zhang G, Wang J, Xie K, Xue Q, Fan X: Analysis of long noncoding RNA and mRNA using RNA sequencing during the differentiation of intramuscular preadipocytes in chicken. Plos One 2017;12:e0172389.

-26 Chen C, Ridzon DA, Broomer AJ, Zhou Z, Lee DH, Nguyen JT, Barbisin M, Xu NL, Mahuvakar VR, Andersen MR: Real-time quantification of microRNAs by stem-loop RT-PCR. Nucleic Acids Res 2005;33:e179.

-27 Livak KJ, Schmittgen TD: Analysis of relative gene expression data using real-time quantitative PCR and the 2(-Delta Delta C(T)) Method. Methods 2001;25:402-408.

28 Li H, Ma Z, Jia L, Li Y, Xu C, Wang T, Han R, Jiang R, Li Z, Sun G: Systematic analysis of the regulatory functions of microRNAs in chicken hepatic lipid metabolism. Sci Rep 2016;6:31766.

29 Leveille GA: In vitro hepatic lipogenesis in the hen and chick. CBP 1969;28:431-435.

-30 Huang HY, Liu RR, Zhao GP, Li QH, Zheng MQ Zhang JJ, Li SF, Liang Z, Wen J: Integrated analysis of microRNA and mRNA expression profiles in abdominal adipose tissues in chickens. Sci Rep 2015;5:16132.

-31 Kim SY, Kim AY, Lee HW, Son YH, Lee GY, Lee JW, Lee YS, Kim JB: miR-27a is a negative regulator of adipocyte differentiation via suppressing PPARgamma expression. Bioche Biophys Res Commun 2010;392:323-328.

-32 Chen L, Dai YM, Ji CB, Yang L, Shi CM, Xu GF, Pang LX, Huang FY, Zhang CM, Guo XR: MiR-146b is a regulator of human visceral preadipocyte proliferation and differentiation and its expression is altered in human obesity. Mol Cell Endocrinol 2014;393:65-74.

33 Zhuang G, Meng C, Guo X, Cheruku PS, Shi L, Xu H, Li H, Wang G, Evans AR, Safe S: A novel regulator of macrophage activation: miR-223 in obesity-associated adipose tissue inflammation. Circulation 2012;125:2892-2903.

34 Gan S, Huang Z, Liu N, Su R, Xie G, Zhong B, Zhang K, Wang S, Hu X, Zhang J: MicroRNA-140-5p impairs zebrafish embryonic bone development via targeting BMP-2 Febs Lett 2016;590:1438.

-35 Wienholds E, Kloosterman WP, Miska E, Alvarez-Saavedra E, Berezikov E, De BE, Horvitz HR, Kauppinen S, Plasterk RH: MicroRNA expression in zebrafish embryonic development. Science 2005;309:310-311.

-36 Yan X, Zhu Z, Xu S, Yang L, Liao XH, Zheng M, Yang D, Wang J, Chen D, Wang L: MicroRNA-140-5p inhibits hepatocellular carcinoma by directly targeting the unique isomerase Pin1 to block multiple cancer-driving pathways. Sci Rep 2017;7:45915.

-37 Zhang X, Chang A, Li Y, Gao Y, Wang H, Ma Z, Li X, Wang B: miR-140-5p regulates adipocyte differentiation by targeting transforming growth factor- $\beta$ signaling. Sci Rep 2015;5:18118.

-38 Nagy L, Kao HY, Love JD, Li C, Banayo E, Gooch JT, Krishna V, Chatterjee K, Evans RM, Schwabe JW: Mechanism of corepressor binding and release from nuclear hormone receptors. Genes Dev 1999;13:3209. 